\title{
Osteopetrotic induced pluripotent stem cells derived from patients with different disease-associated mutations by non- integrating reprogramming methods
}

Fatma Visal Okur ${ }^{1,2^{*}}$, Inci Cevher ${ }^{1}$, Cansu Özdemir ${ }^{1}$, Çetin Kocaefe ${ }^{1,3}$ and Duygu Uçkan Çetinkaya ${ }^{1,2}$

\begin{abstract}
Background: Autosomal recessive osteopetrosis is a genetically and phenotypically heterogeneous disease, caused by defects in osteoclast formation and function. The only available treatment is allogeneic stem cell transplantation that has still high morbidity and mortality. The goal of the present study was to generate iPSCs from bone marrowderived MSCs of osteopetrosis patients with three most common mutations by using two different integration-free gene transfer methods and compare their efficiencies. The secondary objective was to select the most appropriate integration-free production method for our institutional iPSC bank using this rare disease as a prototype.

Methods: Two different integration-free gene transfer methods (episomal and Sendai viral vectors) were tested and compared on the same set of patient samples exhibiting three different mutations associated with osteopetrosis. Generated iPSCs were characterized by standard assays, including immunophenotyping, immunocytochemistry, RTPCR, embryoid body, and teratoma assays. Karyotype analyses were performed to evaluate genetic stability.

Results: IPSC lines exhibiting typical ESC-like colony morphology were shown to express pluripotency markers by immunofluorescence staining. Over 90\% of the cells were found positive for SSEA-4 and OCT3/4 and negative/weak positive for CD29 by flow cytometry. Immunohistochemical staining of teratoma and spontaneously differentiated embryoid body sections confirmed their trilineage differentiation potential. All iPSC lines expressed pluripotencyrelated genes. Karyotype analyses were found normal. Direct sequencing of PCR-amplified DNA showed that disease-related mutations were retained in the patient-specific iPSCS.

Conclusion: Generation of iPSC using SeV and episomal DNA vectors have several advantages over other methods like the ease of production, reliability, high efficiency, and safety, which is required for translational research. Furthermore, owing to the pluripotency and self-renewal capacity, patient-specific iPSCs seem to be ideal cell source for the modeling of a rare genetic bone disease like osteopetrosis to identify osteoclast defects, leading to clinical heterogeneity in osteopetrosis patients, especially among those with different mutations in the same gene.
\end{abstract}

Keywords: Osteopetrosis, Sendai virus, Episomal vector, Mesenchymal stromal cells, Induced pluripotent stem cells, Reprogramming

\footnotetext{
* Correspondence: fvokur@hacettepe.edu.tr

${ }^{1}$ Hacettepe University, Center for Stem Cell Research and Development PEDI-STEM, Ankara, Turkey

²Division of Pediatric Hematology and Bone Marrow Transplantation Unit,

Department of Pediatrics, Faculty of Medicine, Hacettepe University, Ankara,

Turkey

Full list of author information is available at the end of the article
}

(c) The Author(s). 2019 Open Access This article is distributed under the terms of the Creative Commons Attribution 4.0 International License (http://creativecommons.org/licenses/by/4.0/), which permits unrestricted use, distribution, and reproduction in any medium, provided you give appropriate credit to the original author(s) and the source, provide a link to the Creative Commons license, and indicate if changes were made. The Creative Commons Public Domain Dedication waiver (http://creativecommons.org/publicdomain/zero/1.0/) applies to the data made available in this article, unless otherwise stated. 


\section{Highlights}

- Malignant infantile osteopetrosis (MIOP) is a rare genetic bone disease of childhood with no available treatment except allogeneic hematopoietic stem cell transplantations.

- Since MIOP is phenotypically and genotypically heterogeneous disease, there is still a great need for new research tools to identify the factors leading to defective osteoclastogenesis and its impact on bone metabolism and establishment of bone marrow hematopoietic niche.

- Patient-derived iPSCs carrying the disease-causing mutation would be a valuable research tool to study disease pathogenesis within the patient's own genomic background, compared to animal models.

- Clinically relevant patient-specific iPSCs derived by integration-free reprogramming methods, like $\mathrm{SeV}$ and episomal vectors, will pave the way for disease modeling and discovery of new therapeutic targets, which will be of significant value to researchers and clinicians.

\section{Background}

Osteopetrosis is a rare inherited disease characterized by increased bone mass and density resulting from defects in osteoclast formation and/or function $[1,2]$. The disease is classified into two types based on the mode of transmission: autosomal dominant osteopetrosis (ADO) and autosomal recessive osteopetrosis (ARO) [3, 4]. ARO is also termed as "malignant infantile osteopetrosis" (MIOP) which presents soon after birth and often lethal unless treated accordingly [5]. A dysfunction of osteoclasts leads to bone resorption defects and increased bone density, decreased bone strength with abnormal bony overgrowth, and a bone marrow cavity insufficient to support hematopoiesis. Insufficient hematopoiesis results in bone marrow failure presented as severe cytopenias and compensatory extramedullary hematopoiesis. Other clinical manifestations resulting from bony growth are cranial nerve dysfunctions, nasal obstruction, and gross motor developmental delays. Osteoclasts are derived from hematopoietic stem cells, thus the establishment of an allogeneic graft and differentiation of functional osteoclasts after transplantation help to alleviate bone remodeling and recovery from pancytopenia as well as extramedullary hematopoiesis. Even though hematopoietic stem cell transplantation (HSCT) is the only curative treatment option, there is a high risk of graft failure and severe transplant-associated complications [1]. Disease modeling using patient-derived induced pluripotent stem cells (iPSCs) as an unlimited source of autologous cells would help to better understands the disease biology within patient's own genomic background compared to animal models and paves the way for the development of new cell replacement therapies.

Generation of iPSCs is a milestone in stem cell research due to their unlimited maintenance capacity. iPSCs are excellent research tools to study disease modeling, drug screening, modifier gene discovery, and test novel therapeutic approaches including gene and cell replacement in a wide spectrum of inherited diseases or diseases of single gene origin or complex nature $[6,7]$. Patient-specific iPSCs provide unique opportunities for both stem cell biologists and clinicians to dissect the pathogeneses and identify new alternative therapeutic strategies for rare genetic diseases by providing a virtually unlimited source of cells carrying the disease-causing mutations.

Gene delivery is crucial for the generation of iPSCs. To date, several alternatives have been developed and tested. Retroviral systems are characterized by high efficiency and reproducibility even in somatic cells that are difficult to reprogram. But they have some serious limitations such as stable expression of targeted transgenes, some of which are protooncogenes (KLF4 and c-MYC) and the possibility of insertional mutagenesis $[8,9]$. For a successful translation of human iPSCs-derived products into the clinic as gene/cell therapies or other regenerative medicine applications, the safety of manufacturing strategy matters as much as its efficiency. Therefore today, integration-free methods for the generation of iPSCs are in focus [10, 11]. Somatic cell reprogramming can be achieved through various non-integrating methods such as mRNAs $[12,13]$ or proteins [14] or gene transfer through vectors including Sendai virus $(\mathrm{SeV})$ or episomal DNA vectors [15] while avoiding the risk of insertional mutagenesis [16]. However, reprogramming efficiency using either proteins or mRNAs is still very low, and these methods require repetitive delivery of multiple proteins or mRNAs into the somatic cells. Considering the need for reprogramming technologies with higher efficiency and better safety profile for translation into the clinic, episomal vectors and Sendai viral vector which both are non-integrative are accepted to be good alternatives [17, 18]. Here, we used human bone marrow-derived mesenchymal stromal cells as the cell of origin, since they can be isolated and expanded easily from both donors and patients irrespective of their age, with a high proliferative potential. Also, they are multipotent progenitor cells, which may provide further reprogramming advantage considering the stemness of the parenteral cell $[19,20]$.

This study aims to generate iPSCs from bone marrowderived MSCs of osteopetrosis patients by using two different integration-free gene transfer methods (episomal and Sendai viral vectors) and compare the efficiency of two methods in order to select the most appropriate production method. The ultimate aim of this study is use patient-derived IPS cells as a research tool to investigate 
the factors leading to clinical heterogeneity (as well as the discovery of modifier factors and genes) in osteopetrosis patients, especially among those with different mutations in the same gene. This might provide new insights into abnormal osteoclastogenesis and its role in the establishment of bone marrow hematopoietic stem cell niche in this rare genetic bone disease.

\section{Methods}

Isolation of mesenchymal stromal cells, cell culture, and characterization

Mesenchymal stromal cells (MSC) were isolated by density gradient separation from the bone marrow of two healthy donors and three patients with three different ARO associated mutations in CLCN7, TCIRG1, and SNX10 (Additional file 2: Table S1). The enriched mononuclear cell population was then cultured in complete culture medium consisting of DMEM-LG (Gibco) medium supplemented with 10\% FBS, 35\% MCDB (Sigma), $0.1 \%$ L-glutamine at $37{ }^{\circ} \mathrm{C}$ in a $5 \% \mathrm{CO}$. Following $48 \mathrm{~h}$ of culture, cell attachment and morphology were evaluated. After reaching 80-90\% confluency, MSCs were passaged. MSCs were characterized by flow cytometry (positive for CD29, CD44, CD73, CD90, and CD105 and negative for CD34 and CD45). The data were analyzed using BD FACS Diva Software v6.1.2, and the expression of each CD markers on the cells was calculated based on the percentage (Additional file 3: Table S2). Adipogenic and osteogenic differentiation potentials were evaluated for each established cell line. For induction of adipocyte differentiation, cells were treated with adipogenic differentiation medium (DMEM LG (Gibco) supplemented with 10\% FBS (Gibco), $1 \mu \mathrm{M}$ dexamethasone (Sigma), $60 \mu \mathrm{M}$ indomethacin (Sigma), $500 \mu \mathrm{M} 1$ methyl-3-isobutylxanthine (IBMX, Sigma), and $5 \mu \mathrm{g} / \mathrm{ml}$ insulin (Sigma-Aldrich) for 21 days. Cells were fixed and stained with Oil Red O stain to visualize fat droplets in the cells. For osteoblastic differentiation, cells were treated with osteoblast-induction media $(\mathrm{OB})$ containing DMEM-LG (Gibco) supplemented with 10\% FBS (Gibco), $100 \mathrm{nM}$ dexamethasone (Sigma), $10 \mathrm{mM}$ beta glycerophosphate (Sigma), and $0.2 \mathrm{mM}$ ascorbic acid (Sigma) for 21 days. Cells were fixed and stained with Alizarin red. The preparations of stained cells were visualized using an IX73 microscope (Olympus).

\section{Reprogramming of mesenchymal stromal cells}

MSCs were reprogrammed by two different vector systems. A mixture of three Sendai virus-based $(\mathrm{SeV})$ vectors expressing a classic set of reprogramming transgenes Oct4, Sox2, Klf4, and c-Myc (CytoTune-iPS Sendai Reprogramming Kit, A1378001, Invitrogen) (29) and an episomal vector system (Epi5) containing an optimized mixture of three oriP/EBNA-1(Epstein-Barr nuclear antigen-1)-based episomal plasmids (30). The latter approach uses Oct4, Sox2, Lin28, L-Myc, and Klf4 as the transgenes for reprogramming and also utilize two additional plasmids expressing mp53DD (dominantnegative mutation of $\mathrm{p} 53$ protein) and EBNA1 to facilitate episomal plasmid DNA replication in dividing cells to enhance reprogramming efficiency (Epi5, Episomal iPSC Reprogramming Kit, A15960, Invitrogen) [21, 22].

\section{SeV reprogramming}

One day prior to transduction, early passage (P3-P4) MSCs were harvested with Accutase, plated in a 6-well plate coated with $0.1 \%$ gelatine at $1 \times 105$ cell/ well density and maintained in MSC growth medium (10\% FBS, $1 \%$ L-glutamine (2 mM), low-glucose (3.5 g/dl) DMEM). Next day, 80-85\% confluent MSCs were transduced with $\mathrm{SeV}$ vectors at MOI of 5:3:3 in $2 \mathrm{ml}$ fresh MSC medium for $24 \mathrm{~h}$. At day 5, the cells were harvested with Accutase, counted and seeded in a Matrigel-coated $10 \mathrm{~cm}$ dish at $1 \times 105$ cells/well confluence and switched into TeSRTM-E7TM reprogramming medium (Stemcell Technologies). Cells were maintained in TeSRTME8TM (Stem Cell Technologies) medium starting from day 14. IPS colonies that started to appear around day 18 were harvested with manual microdissection method, transferred into Matrigel-coated dishes, and were expanded using EDTA clump passaging approach [23].

\section{Episomal reprogramming}

Early passage (P3-P4) MSCs at 80-85\% confluence were harvested with Accutase, and $1 \times 106$ cells were resuspended in nucleofector solution supplied in the P1 Primary Cell 4D-Nucleofector ${ }^{\oplus} \mathrm{X}$ Kit L (Lonza). One microliters of an optimized mixture of Epi5 TM reprogramming plasmids (Oct4, Sox2, Klf4, L-Myc, and Lin28) and $1 \mu \mathrm{l}$ of an optimized mixture of mP53DD and EBNA1 plasmids were added to the cell suspensions. pMax-GFP was used as a transfection control plasmid. The cell suspension was transfected by using program FF-104 on a 4D-NucleofectorTM X Unit. In order to assess the transfection efficiency, GFP expression on transfected MSC cells was checked by both immunofluorescence microscopy (Olympus-IX73) and flow cytometry $24 \mathrm{~h}$ later. Following the identification of the IPS colonies, cells were harvested and maintained as described above.

Reprogramming efficiencies of both methods were determined by alkaline phosphatase (AP) staining of the whole plate (described below) at around day 24-28 posttransduction/post-transfection when colonies were ready for picking. The reprogramming efficiency was defined as the ratio of the number of colonies positive for alkaline phosphatase activity to the total number of cells used in the reprogramming experiment. 


\section{Characterization of iPSC lines}

Besides colony morphology and alkaline phosphatase activity, immunofluorescence staining, flow cytometry, and karyotype analyses were performed for the characterization of selected IPS lines. Expression of pluripotency genes and detection of residual $\mathrm{SeV}$ and episomal plasmid sequences were evaluated in reprogrammed IPS cells using real-time PCR and conventional PCR. Embryoid body formation and teratoma assays were performed to assess pluripotency in vitro and in vivo. As per each sample, three IPS lines were characterized.

Alkaline phosphatase activity of iPSCs was determined by staining with Blue AP Staining Kit vector labs SK530. Immunofluorescence staining was done using PSC 4-Marker Immunocytochemistry Kit (Life Technologies). In brief, after fixation and permeabilization, IPSC colonies were first incubated with primary antibodies (OCT4, SSEA4, SOX2, and TRA1-60) for $3 \mathrm{~h}$, and then with conjugated secondary antibodies for $1 \mathrm{~h}$ at RT. Following staining with DAPI, samples were examined on a fluorescence microscope (Olympus- IX73).

Expression of pluripotency markers on iPS cells was evaluated with flow cytometry (FACS Aria and Navios EX Beckman Coulter). iPSC colonies were harvested with Accutase, and single cell suspensions were stained using antibodies against SSEA4, OCT4, and CD29 (Additional file 3: Table S2). Gates on flow cytometry were defined according to isotype controls.

Genetic stability of iPSC cell lines was evaluated by standard Giemsa-banded karyotype analysis. Verification of the retained mutations was assessed by sequencing of the genomic locus.

The mRNA expression levels of the pluripotency genes (Endo-Oct4, Endo-Sox2, Nanog, c-Myc, Klf-4, Utf-1, Dnmt3b, Tert-1, Rex-1, CDH-1,) were analyzed by reverse transcriptase coupled quantitative real-time PCR (qRT-PCR). Total RNA isolation was conducted by Promega, ReliaPrep ${ }^{\text {ma }}$ RNA Cell Miniprep System (Cat no: Z6012). qPCR studies were performed using ThermoFisher Maxima SYBER Green/ROX qPCR master mix on a Rotorgene 6000 (Corbett Life Science, Australia) fluorometric PCR instrument. Gene expression was normalized to the expressions of $\beta$-actin and TFIID as reported previously [24]. The losses of Sendai virus genome ( $\mathrm{SeV}$ genome sequence targeted) and episomal DNA (OriP sequence targeted) were assessed by endpoint PCR using Promega GoTaq ${ }^{\circ}$ DNA polymerase (Additional file 3: Table S2). Agarose gel electrophoresis results of end-point PCR analysis of cDNA expression were presented.

In vitro trilineage differentiation potential of iPSC lines was evaluated with embryoid body (EB) assay. iPSC colonies were harvested using Accutase, single-cell suspensions were prepared at passage 15-20, and cells were seeded onto AggreWellTM800 plates (StemCell Technologies). Following a 24-h suspension culture, EBs were harvested and transferred to ultra-low attachment plates in STEMdiffTM APELTM 2 Medium (StemCell Technologies) and kept in culture for spontaneous differentiation. Differentiated EBs were stained with hematoxylin and eosin. Microsections of paraffin-embedded EBs were also stained with antibodies against OCT4, vimentin, and synaptophysin.

In vivo trilineage differentiation potential of iPSCs was demonstrated with teratoma assay. iPSCs (passages 1520) in six-well plates were dissociated manually. iPSCs were injected subcutaneously into 3-month-old female $\mathrm{Blb} / \mathrm{C}$-Nude mice. Around 8-week post-injection terotoma formation was observed, mice were sacrificed and teratoma was dissected. Immunohistochemical stains for hematoxylin and eosin as well as Mallory trichrome and Toluidine Blue were performed for the evaluation of lineage-specific differentiation.

\section{Statistical analysis}

$P$ values were calculated using chi-square test and method-specific efficiencies were provided as geometric means \pm SEM. Arbitrary gene expression levels were achieved by normalizing the gene of interest to the geometrical mean expressions of reference genes as described previously [24]. The arbitrary gene expression was further normalized to the mean expression of control samples to achieve fold change values. Analysis of variance was conducted on the replicate values of experiment groups, and the groups satisfying statistical significance were indicated where appropriate $(*)$. The data was analyzed using $\mathrm{R}$ statistical package (version 3.5.1) using BRB Array Tools interface (version 4.6.0).

\section{Results}

Bone marrow-derived mesenchymal stromal cell culture and characterization

BM-MSCs were characterized by morphology, immunophenotyping, and evaluation of differentiation potential. Mycoplasma testing proved that all MSC lines were free from contamination. Flow cytometry analyses showed that all tested MSCs expressed specific surface markers of mesenchymal stromal cells, CD29, CD44, CD73, $\mathrm{CD} 90$, and CD105, and they were found negative for CD34 and CD45. Mesodermal lineage differentiation potential of BM-MSCs was assessed by induction of adipogenic and osteogenic differentiation (Additional file 1: Figure S1).

Generation, expansion, and characterization of iPSC lines from BM-MSCs of osteopetrosis patients

We performed both $\mathrm{SeV}$ - and Epi5-mediated inductions from three patients who had disease-associated mutations 
in three different genes (TCIRG1, SNX10, and CLCN7) representing the diverse genetic heterogeneity of osteopetrosis phenotype and two healthy donors, under the same culture conditions. Three IPS lines per sample for each method were selected and characterized using the standard pluripotency assays. Reprogramming efficiencies were calculated and loss of $\mathrm{SeV}$ genome and Episomal plasmid were tested to understand the time-frame of the loss of episomal agents along with iPSC expansion. Finally, verification of the mutations was assured for all IPSC lines derived from osteopetrosis patients.

Transfection efficiency of episomal plasmids in BMMSCs was evaluated by EGFP expression and was found to be $72 \% \pm 3.46$. The first ES- like colonies were observed at around day 18 post-transduction with $\mathrm{SeV}$ and around day 21 post-transfection with episomal plasmids. For each patient's line, 8-10 iPSC colonies were picked manually and further expanded in culture. Control plates were stained with alkaline phosphatase to identify iPSC colonies, and the mean efficiencies of successful reprogramming experiments of osteopetrotic BM-MSCs were found as $0.3 \pm 0.11 \%$ for $\mathrm{SeV}$ and $0.1 \pm 0.02 \%$ for Epi5 method, higher than those of the control $(0.14 \pm 0.01 \%$ for $\mathrm{SeV}$ and $0.07 \%$ for Epi5).

All hiPSC lines derived from osteopetrosis patients expressed alkaline phosphatase and other stemness markers (SSEA4, OCT3/4, TRA1-60, SOX2) as revealed by immunofluorescence staining, flow cytometry (SSEA4, OCT3/4) (Fig. 1a), and qRT-PCR (Fig. 2). Flow cytometry analyses demonstrated that more than $90 \%$ of SeV- and Epi5-derived iPSCs were positive for SSEA4 (SeV $97.21 \% \pm 0.69$; Epi5 $99 \% \pm 0.11 P>0.05)$ and OCT3/4 positive ( $\mathrm{SeV} 94.09 \% \pm 0.63$; Epi5 $94 \% \pm 1.07 P>0.05$ ), while they were either negatively or weakly positive for CD29 (SeV 1.6\% \pm 0.36; Epi5 2.7\% $\pm 0.81 P>0.05$ ) (Fig. 1b). Karyotype analyses were found normal for all iPSC lines (Fig. 1c). Mutation verification showed that diseaserelated mutations were retained in the iPSCs.

The analyses of pluripotency gene expression panel consisted of ten different target mRNA. Out of the four transgenes (OSKM factors) that were utilized for reprogramming, OCT4 and SOX2 were differentially assessed to delineate endogeneous versus exogenic (transgene) expression, since the timing and the levels of OCT4 and SOX2 expression are important indicators of reprogramming efficiency [25]. The cumulative expressions of other investigated transcripts (KLF4, c-MYC, NANOG, REX1, TERT1, DNMT3b, CDH1, UTF1) were presented (Fig. 2).

The pluripotency gene expressions were evaluated at different time points (passages 5-20) for each established iPSC lines. Results revealed that the induction of pluripotency was already achieved at P5 and none of the investigated genes exhibited any significant time-course variance along the observed period for IPSC lines obtained with both methods. The major observed difference is the absence of the expression of investigated genes in a negative control sample (primary BM-MSC) excluding KLF4 [26], (Fig. 2). In this regard, the presence of the expression of these genes discriminate samples from negative controls in an on/off manner. Next, we tested how quickly the exogenous reprogramming agents were lost during iPSC expansion. We observed a passage-dependent decrease in both SeV RNA and episomal DNA levels in all iPSC lines, but an accelerated loss of SeV RNA in all the patient-iPSC lines and all $\mathrm{SeV}$ patients IPS lines were negative by P5. The retention of the episomal genome in both Epi5 and $\mathrm{SeV}$ induced IPS colonies derived from the same control is probably sample-dependent and may be related to epigenetic and proliferative features of the donor BM-MSCs (Fig. 3).

Finally, in vitro trilineage differentiation potential of generated hiPSC lines were evaluated with EB formation and ability of iPSC-derived EBs to form representatives of three germ layers. Uniform size- and spheroid-shape EBs were maintained in suspension culture to induce spontaneous differentiation up to 21 days before immunohistological analyses (Fig. 4a). Morphological change similar to early embryogenesis such as increase in size, 3D spheroidal appearance, development of an inner cell layer and formation of cystic areas were observed over time. Hematoxylin and eosin-stained histologic sections of spontaneously differentiated derivatives showed trilineage potential. Expression of neuroectodermal (synaptophysin) and mesodermal (vimentin) markers in the differentiated EBs, together with lack of pluripotency marker (OCT3/4) confirmed their differentiation potential (Fig. 4b).

In vivo trilineage differentiation potential of patientspecific iPSCs was demonstrated by teratoma formation assay. Histological examination of the teratomas revealed the presence of a set of representative tissues that were originated from the three embryonic germ layers, including epithelium, muscle, connective tissue, peripheral nerve, and central nervous system (Fig. 4c).

Taken together, our results demonstrate that iPSCs could be derived efficiently from osteopetrosis patients with different disease-associated mutations, using two different integration-free reprogramming methods. There was not any significant qualitative difference among the iPSC lines obtained with compared two methods.

\section{Discussion}

Autosomal recessive osteopetrosis is a genetically and phenotypically heterogeneous disease, which is caused by defects in osteoclast formation and function. Although seven genes have been identified so far as having a role in disease pathogenesis, a study on disease-associated mutations continues 


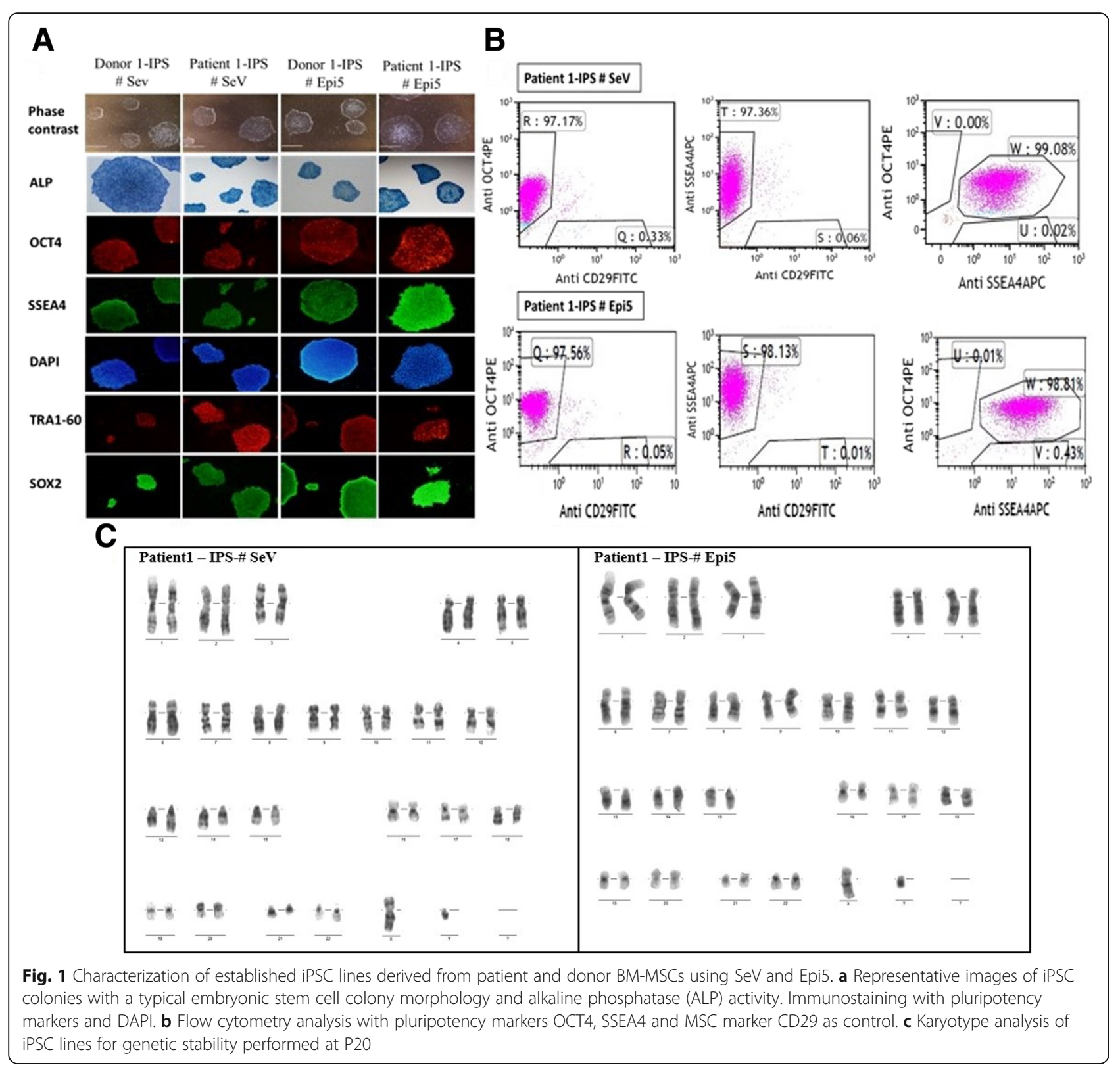

to discover essential genes for osteoclast function and to better understand osteoclast biology. Use of patient-derived pluripotent stem cells could be an alternative to genetically modified cell lines or animal models for these functional studies, providing a virtually unlimited source of autologous cells carrying the disease-causing mutations. In this study, we derived and expanded iPSC lines successfully from osteopetrosis patients with mutations in TCIRG1, CLCN7 and SNX10 genes (accounting nearly 70\% of all cases) using two different integration-free reprogramming methods under feeder-free culture conditions.

Both $\mathrm{SeV}$ and Epi5 reprogramming methods were found quite efficient and highly reliable for the generation of patient-specific iPSCs from BM-MSCs. Characterizations confirmed that the generated iPSCs expressed pluripotent stem cell markers, showed trilineage differentiation potential, displayed normal karyotype and retained the diseaseassociated mutations.

We evaluated the acquisition of the pluripotency state through the investigation of several pluripotency-related transcripts in iPSC lines at selected time points. Excluding some of the investigated genes, these data did not exhibit any striking variance in a time course manner. The observed variations for some of the investigated transcripts may reflect the natural fluctuation of gene expression in between samples, which may be secondary to the epigenetic state of the reprogrammed cell genome as well as the exposure to the reprogramming factors. 


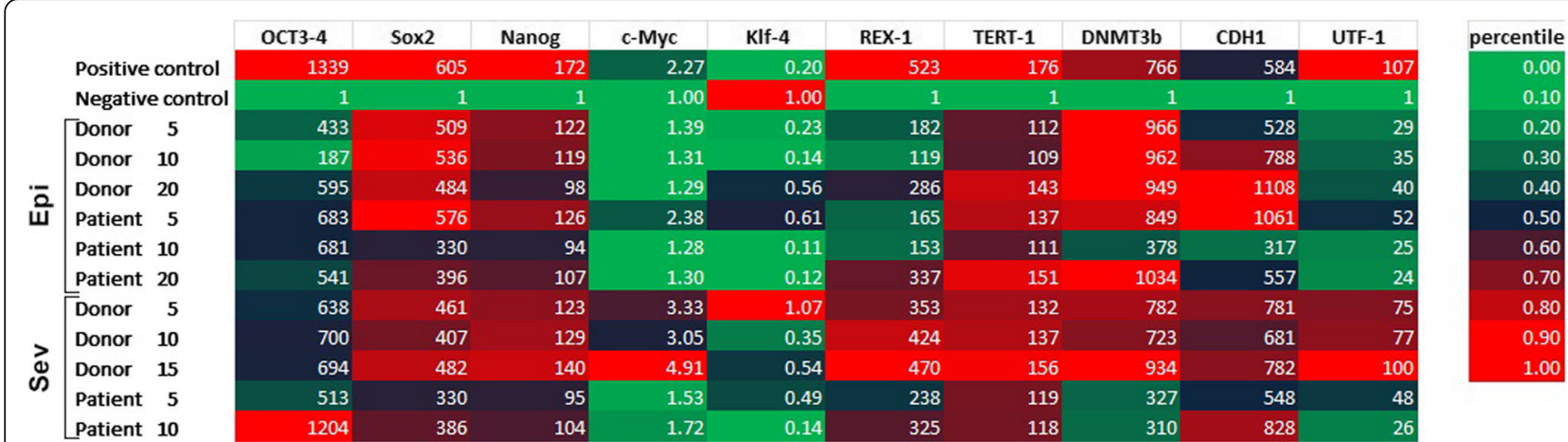

Fig. 2 Relative expression of pluripotency genes in established iPSC lines. Relative arbitrary expression values are normalized to the negative control sample and indicated as fold change. Endogenous OCT4 and SOX2 expressions were analyzed differentially to demarcate any expression originating from the transgenes. The cumulative expressions were given for all the other genes. The expressions of pluripotency genes were detectable in all of the tested lines for all time-points, with no significant variations between the lines $(n=47$ iPSC lines, three lines from each sample at different time points; P5-P20)

The variant clustering of KLF4 and cMyc genes among control BM-MSCs may be related to the nature of these transcripts in BM-MSCs, but may not exhibit a similar pattern in iPSC reprogramming of a cell from any other somatic source [26]. These data show that iPSC reprogramming is an on/off phenomenon that would remain unchanged over time once the culture conditions are maintained standard, independent from the method of reprogramming. Similarly, Trevisan et al. who investigated the impact of the reprogramming methods (retrovirus, $\mathrm{SeV}$, and episomal) on the quality of iPSCs identified no significant difference among the stem cell gene expression profiles of iPSCs derived with different methods [27].

Our results support the study of Daley et al. comparing three different non-integrating reprogramming methods (SeV, Episomal, and mRNA) using a number of criteria [21]. They reported the reprogramming efficiencies as $0.077 \%$ for $\mathrm{SeV}$ and $0.013 \%$ for Epi5. Higher efficiencies in our experiments for both methods compared to the healthy donor BM-MSCs could be attributed to primary disease-specific and/or parenteral cell-specific features that make BM-MSCs more prone to reprogramming. They observed a passage-dependent decrease in viral load over time in $\mathrm{SeV}$-mediated iPSC lines which occurred at later passages, relative to episomal sequences. We also observed a passage-dependent decrease in reprogramming agents in all of the iPSC lines and complete loss at higher passages, but an accelerated loss of SeV RNA in all the patient-iPSC lines. The retention of episomal genome is probably sample-dependent
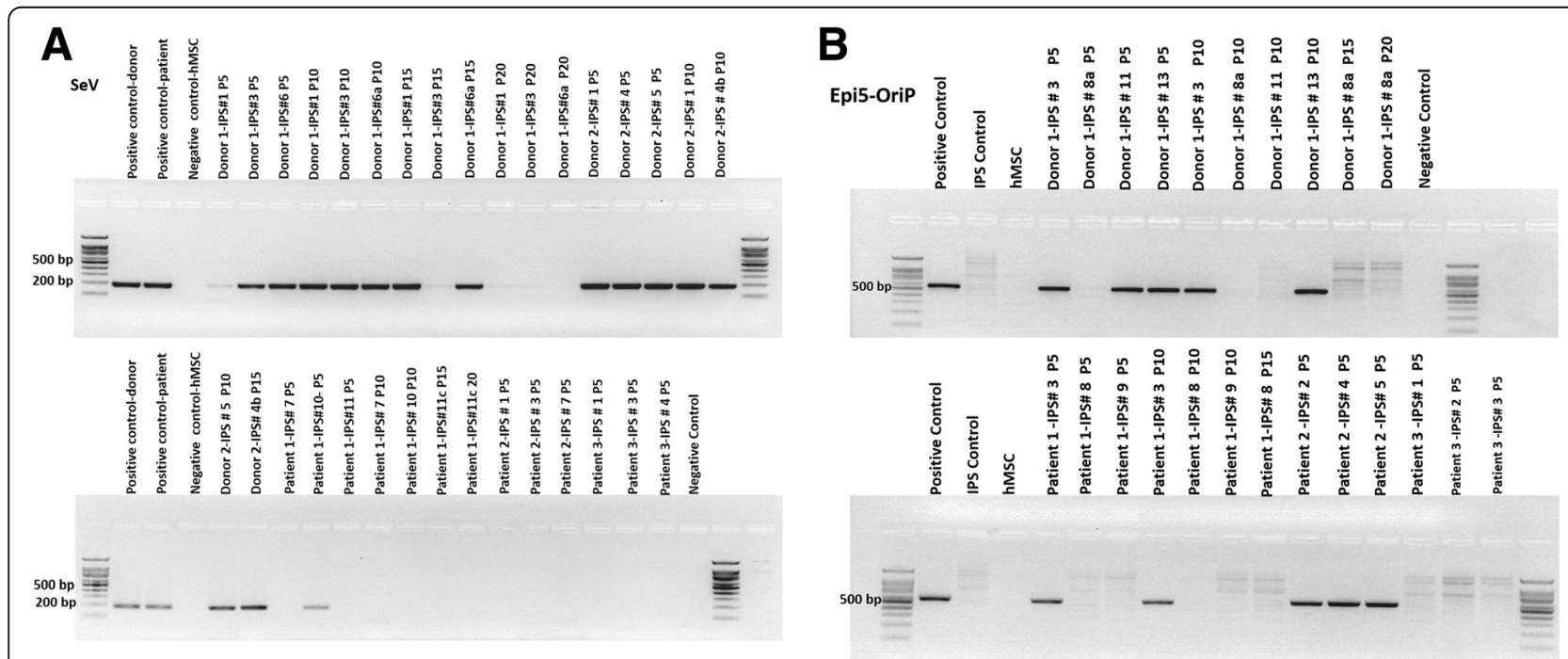

Fig. 3 a, b Retention of reprogramming agents. SeV and Epi5 derived IPS lines show different dynamics of the loss of viral genome (a) and episomal plasmid (b) 


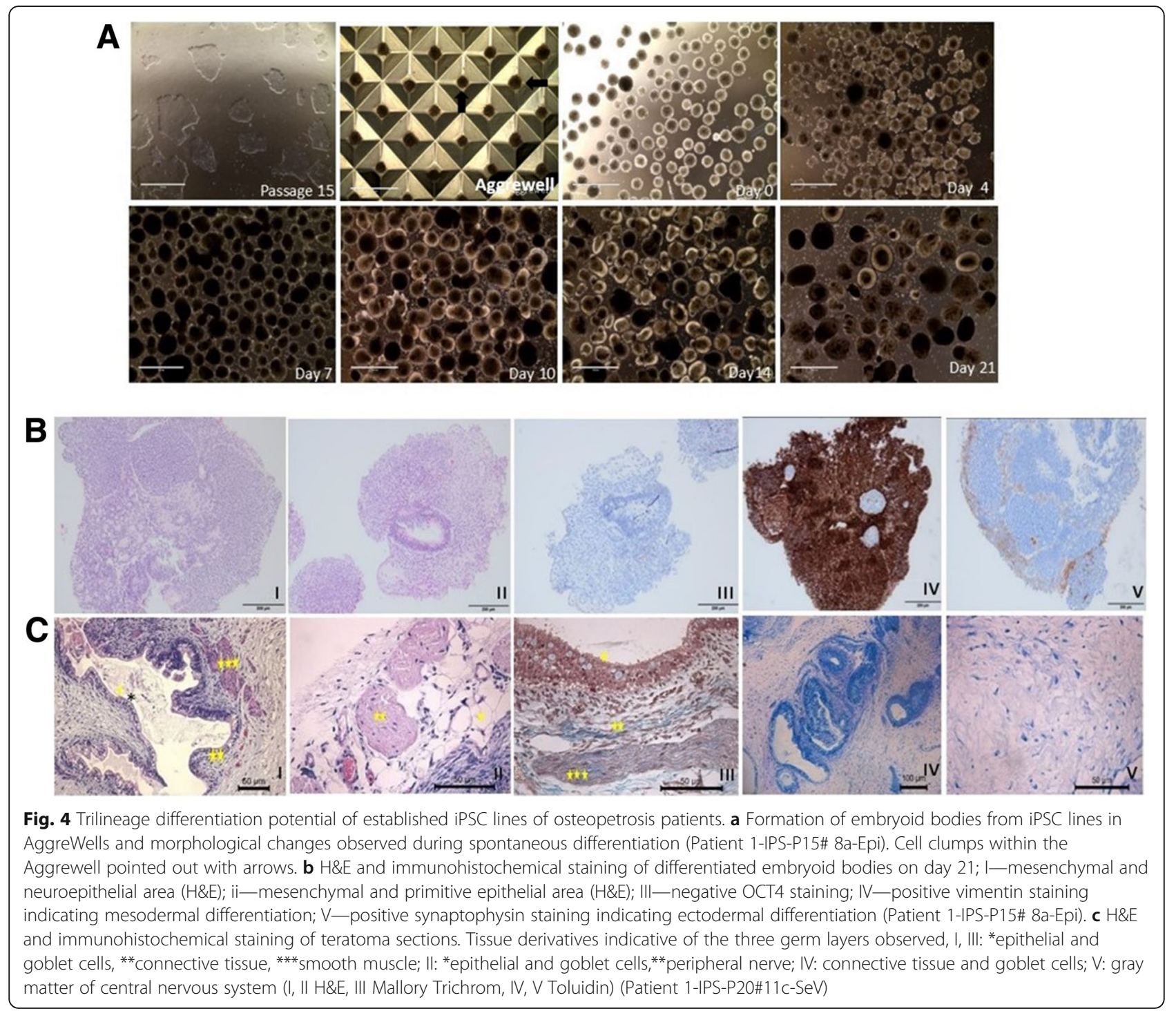

and may be related to epigenetic and proliferative features of the donor BM-MSCs.

In another study evaluating the integration-free reprogramming methods for obtaining clinically relevant iPSCs, there were not any qualitative differences in iPSC lines, except reprogramming efficiencies [16]. It seems that major determinants of reprogramming success are sample-related factors, cell of origin (proliferation and differentiation status of cell and cellular epigenetic features), and culture conditions rather than the method itself [28]. A range of somatic cell types including neonatal fibroblasts, dental pulp cells, adipose cells, CD34+ cells from the umbilical cord, and peripheral blood mononuclear cells has been successfully reprogrammed with $\mathrm{SeV}$ and Episomal methods [11, 17, 29, 30]. We preferred to reprogram BM-MSC of osteopetrosis patients, because of their multipotency, ease of purification/expansion and wellestablished characteristics [19, 20]. As we hypothesized
iPSC lines derived readily from BM-MSCs of all patients and healthy donors with high efficiency. BM-MSC-derived osteopetrotic iPSCs would also be a valuable research tool to investigate defective osteoclastogenesis and interaction between osteoclasts and hematopoietic niche, considering phenotypical heterogeneity of patients.

Both $\mathrm{SeV}$ and Episomal vectors allow expression of transgenes without risk of host genome modification. Reprogramming efficiency of $\mathrm{SeV}$ method is higher than that by other methods, especially without using any small molecules. Also, it is possible to select iPSCs that depleted viral genome using an antibody against viral $\mathrm{HN}$ protein expressed on surface of infected cells. Episomal plasmids, on the other hand, can be manufactured and qualified for GMP use at a lower cost. Reprogramming efficiency of episomal plasmids can be boosted further using epigenetic modifiers or other reprogramming factors. Thus, the choice of reprogramming method should be dependent 
on applications in which generated iPSC lines will be used such as in vitro stem cell research or clinical translation.

\section{Conclusion}

In conclusion, we reprogrammed bone marrow-derived MSCs of osteopetrosis patients with three different osteopetrosis-associated mutations in three different genes by using two different integration-free gene transfer methods (episomal and Sendai viral vectors) and the efficiency of two methods were compared to select the most appropriate production method. There were not any method-specific differences in the expression levels/ patterns of pluripotency markers and the developmental potential in generated iPSC lines.

Patient-derived iPSCs would provide limitless cell source to study osteoclast defects associated with diseasespecific mutations within the context of a patient's whole genome. This is important for the understanding of the factors leading to clinical heterogeneity in osteopetrosis patients especially among those with different mutations in the same gene. These investigations will also pave the way for identification of new therapeutic targets, testing new drugs and development of genetically modified cell therapy products.

\section{Additional files}

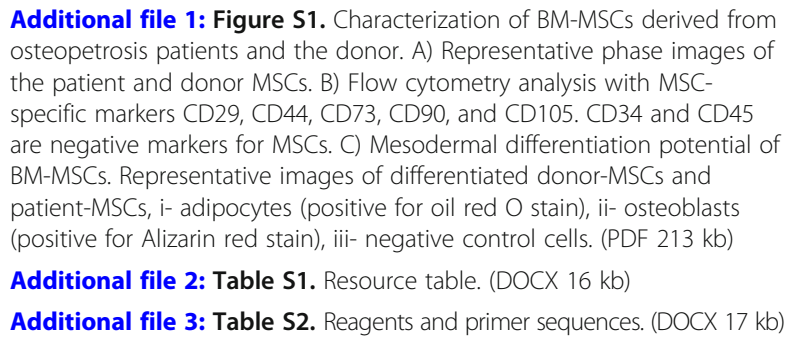
osteopetrosis patients and the donor. A) Representative phase images of the patient and donor MSCs. B) Flow cytometry analysis with MSCspecific markers CD29, CD44, CD73, CD90, and CD105. CD34 and CD45 are negative markers for MSCs. C) Mesodermal differentiation potential of BM-MSCs. Representative images of differentiated donor-MSCs and patient-MSCs, i- adipocytes (positive for oil red O stain), ii- osteoblasts (positive for Alizarin red stain), iii- negative control cells. (PDF 213 kb)

Additional file 2: Table S1. Resource table. (DOCX 16 kb) Additional file 3: Table S2. Reagents and primer sequences. (DOCX $17 \mathrm{~kb}$ )

\footnotetext{
Abbreviations

ADO: Autosomal dominant osteopetrosis; AP: Alkaline phosphatase: ARO: Autosomal recessive osteopetrosis; BM-MSC: Bone marrow mesenchymal stem cell; c-MYC: c-MYC binding protein (MYCBP) pseudogene; $\mathrm{CDH1}$ : Cadherin 1; CDNA: Complementary DNA; CLCN7: Chloride voltage-gated channel 7; DAPI: 4',6-Diamidio-2-phenylindole dihydrocloride; DMEM-LG: Dulbecco's modified Eagle medium; DNA: Deoxyribonucleic acid; DNMT3B: DNA methyltransferase 3 beta; EB: Embryoid body; EBNA-1: Epstein-Barr nuclear antigen-1; EDTA: Ethylenediaminetetraacetic acid; EGFP: Enhanced green fluorescent protein; Endo-Oct4: Endogenous OCT4; Endo-Sox2: Endogenous SOX2; Epi5: Episomal Vector System; ESC: Embryonic stem cell; FACS: Fluorescenceactivated sorting; FBS: Fetal bovine serum; GFP: Green fluorescence protein; GMP: Good manufacturing practice; H\&E: Hematoxylin and eosin; HSCT: Hematopoietic stem cell transplantation; IBMX: 1-Methyl-3isobutylxanthine; iPSCs: Induced pluripotent stem cells; KLF4: Kruppel like factor 4; MIOP: Malignant infantile osteopetrosis; MOI: Multiplicity of infection; MSCs: Mesenchymal stem cell; mRNA: Messenger RNA; NANOG: Nanog Homeobox; OCT3/4: Octamer-binding transcription factor 4; PCR: Polymerase chain reaction; REX1: RNA exonuclease 1 homolog pseudogene; RNA: Ribonucleic acid; RT-PCR: Real-time PCR; qPCR: Quantitative PCR; qRT-PCR: Quantitative real-time PCR; SEM: Standard error of the mean; SeV: Sendai viral vector; SNX10: Sorting nexin 10;
}

SOX2: SRY-box 2; SSEA-4: Stage-specific embryonic antigen-4; TCIRG1: T cell immune regulator 1, ATPase $\mathrm{H}+$ transporting V0 subunit a3;

TERT: Telomerase reverse transcriptase; UTF1: Undifferentiated embryonic cell transcription factor 1

\section{Acknowledgements}

We are thankful to Jean J. Kim, PhD (Director), for providing expertise and Human Stem Cell Core of Baylor College of Medicine for continuous technical support. We also thank Dr. Petek Korkusuz and Dr. Aytekin Akyol for their assistance with H\&E and immunohistochemical stainings.

\section{Authors' contributions}

FVO and DUC designed the methods and experiments. FVO, IC, CO, and CK performed the experiments. $\mathrm{CO}$ and CK performed bioinformatics analyses. FVO and DUC identified the patients, designed the patient sample collection, and collected the patient samples. All authors approved the final draft and made modifications to the text.

\section{Authors' information}

Not applicable

\section{Funding}

This study is supported by The Scientific and Technological Research Council of Turkey (TUBITAK), 1003-Primary Subjects R\&D Funding Program, Project No: 2135181.

\section{Availability of data and materials}

All data and materials are available in the manuscript.

\section{Ethics approval and consent to participate}

The research was in compliance with the Helsinki Declaration and approved by the Ethics Committee of Hacettepe University. Written informed consents for collection, storage, and use of cells for research purposes were obtained and the study is approved by the Instituonal Review Board of Hacettepe University (Study approval number: 16969557-1213).

\section{Consent for publication}

Not applicable.

\section{Competing interests}

The authors declare that they have no competing interests.

\section{Author details}

${ }^{1}$ Hacettepe University, Center for Stem Cell Research and Development PEDI-STEM, Ankara, Turkey. ${ }^{2}$ Division of Pediatric Hematology and Bone Marrow Transplantation Unit, Department of Pediatrics, Faculty of Medicine, Hacettepe University, Ankara, Turkey. ${ }^{3}$ Department of Medical Biology, Hacettepe University School of Medicine, Ankara, Turkey.

Received: 6 March 2019 Revised: 21 June 2019

Accepted: 30 June 2019 Published online: 17 July 2019

\section{References}

1. Sobacchi C, et al. Osteopetrosis: genetics, treatment and new insights into osteoclast function. Nat Rev Endocrinol. 2013;9(9):522-36.

2. Stark Z, Savarirayan R. Osteopetrosis. Orphanet J Rare Dis. 2009;4:5.

3. Del Fattore A, Cappariello A, Teti A. Genetics, pathogenesis and complications of osteopetrosis. Bone. 2008;42(1):19-29.

4. Perdu B, Van Hul W. Sclerosing bone disorders: too much of a good thing. Crit Rev Eukaryot Gene Expr. 2010;20(3):195-212.

5. Villa A, et al. Infantile malignant, autosomal recessive osteopetrosis: the rich and the poor. Calcif Tissue Int. 2009;84(1):1-12.

6. Pettinato $G$, Wen $X$, Zhang N. Formation of well-defined embryoid bodies from dissociated human induced pluripotent stem cells using microfabricated cell-repellent microwell arrays. Sci Rep. 2014;4:7402.

7. Choi IY, Lim H, Lee G. Efficient generation human induced pluripotent stem cells from human somatic cells with Sendai-virus. J Vis Exp. 2014;(86):1-9.

8. Park HJ, et al. Nonviral delivery for reprogramming to pluripotency and differentiation. Arch Pharm Res. 2014;37(1):107-19.

9. Okita K, Ichisaka T, Yamanaka S. Generation of germline-competent induced pluripotent stem cells. Nature. 2007:448(7151):313-7. 
10. Gonzalez F, Boue S, Izpisua Belmonte JC. Methods for making induced pluripotent stem cells: reprogramming a la carte. Nat Rev Genet. 2011;12(4): 231-42.

11. OKita $\mathrm{K}$, et al. A more efficient method to generate integration-free human iPS cells. Nat Methods. 2011;8(5):409-12.

12. Warren $\mathrm{L}$, et al. Highly efficient reprogramming to pluripotency and directed differentiation of human cells with synthetic modified mRNA. Cell Stem Cell. 2010;7(5):618-30.

13. Miyoshi $\mathrm{N}$, et al. Reprogramming of mouse and human cells to pluripotency using mature microRNAs. Cell Stem Cell. 2011;8(6):633-8.

14. Zhou $\mathrm{H}$, et al. Generation of induced pluripotent stem cells using recombinant proteins. Cell Stem Cell. 2009;4(5):381-4.

15. Yu J, et al. Human induced pluripotent stem cells free of vector and transgene sequences. Science. 2009;324(5928):797-801.

16. Goh PA, et al. A systematic evaluation of integration free reprogramming methods for deriving clinically relevant patient specific induced pluripotent stem (iPS) cells. PLoS One. 2013;8(11):e81622.

17. Okita K, et al. An efficient nonviral method to generate integration-free human-induced pluripotent stem cells from cord blood and peripheral blood cells. Stem Cells. 2013;31(3):458-66.

18. Li HO, et al. A cytoplasmic RNA vector derived from nontransmissible Sendai virus with efficient gene transfer and expression. J Virol. 2000;74(14): 6564-9.

19. Varela I, et al. Generation of human beta-thalassemia induced pluripotent cell lines by reprogramming of bone marrow-derived mesenchymal stromal cells using modified mRNA. Cell Reprogram. 2014;16(6):447-55.

20. Ohnishi $\mathrm{H}$, et al. A comparative study of induced pluripotent stem cells generated from frozen, stocked bone marrow- and adipose tissue-derived mesenchymal stem cells. J Tissue Eng Regen Med. 2012;6(4):261-71.

21. Schlaeger TM, et al. A comparison of non-integrating reprogramming methods. Nat Biotechnol. 2015:33(1):58-63.

22. Drozd AM, et al. Generation of human iPSCs from cells of fibroblastic and epithelial origin by means of the oriP/EBNA-1 episomal reprogramming system. Stem Cell Res Ther. 2015;6:122.

23. Hartung, O., et al., Clump passaging and expansion of human embryonic and induced pluripotent stem cells on mouse embryonic fibroblast feeder cells. Curr Protoc Stem Cell Biol, 2010. Chapter 1: p. Unit 1C 10.

24. Vandesompele J, et al. Accurate normalization of real-time quantitative RTPCR data by geometric averaging of multiple internal control genes. Genome Biol. 2002;3(7):RESEARCH0034.

25. Takahashi K, Yamanaka S. A decade of transcription factor-mediated reprogramming to pluripotency. Nat Rev Mol Cell Biol. 2016;17(3):183-93.

26. Foroutan T. Comparison of differentiation of induced pluripotent stem cells and bone-marrow mesenchymal stem cells to osteoblast: osteogenesis versus pluripotency. Int J Organ Transplant Med. 2016;7(2):91-6.

27. Trevisan $M$, et al. Reprogramming methods do not affect gene expression profile of human induced pluripotent stem cells. Int J Mol Sci. 2017;18(1): 206.

28. Bayart E, Cohen-Haguenauer O. Technological overview of iPS induction from human adult somatic cells. Curr Gene Ther. 2013;13(2):73-92.

29. Choi HY, et al. Generation of a human induced pluripotent stem cell line, KSCBi003-A, from human adipose tissue-derived mesenchymal stem cells using a chromosomal integration-free system. Stem Cell Res. 2018;31:1-4.

30. Chou BK, et al. A facile method to establish human induced pluripotent stem cells from adult blood cells under feeder-free and xeno-free culture conditions: a clinically compliant approach. Stem Cells Transl Med. 2015;4(4): $320-32$

\section{Publisher's Note}

Springer Nature remains neutral with regard to jurisdictional claims in published maps and institutional affiliations.

Ready to submit your research? Choose BMC and benefit from:

- fast, convenient online submission

- thorough peer review by experienced researchers in your field

- rapid publication on acceptance

- support for research data, including large and complex data types

- gold Open Access which fosters wider collaboration and increased citations

- maximum visibility for your research: over $100 \mathrm{M}$ website views per year

At BMC, research is always in progress.

Learn more biomedcentral.com/submissions 